\title{
Robust human-inspired power law trajectories for humanoid HRP-2 robot
}

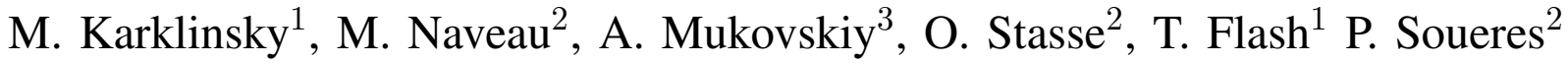

\begin{abstract}
The one-third power law models how human speed of motion depends on the path's curvature. This paper studies the interest of using this law for humanoid robot walking control along a planar reference trajectory. We predicted that humanoid robots following a reference trajectory may benefit from one-third power law speed profiles by reducing closed-loop drift and energy consumption. To robustly execute reference trajectories, we use contracting morphed Andronov-Hopf oscillators, regularized to follow a power law while converging to a planned cyclic trajectory. The walking pattern generator of HRP-2 uses these guiding dynamics to walk along elliptic trajectories. In dynamic simulation, we observe minimal geometric drift with the one-third power law, demonstrating increased precision compared with constant speed and other power laws. Closed-loop experiments on HRP-2 result in a small drift of all power law motions from the reference trajectory, showing the efficiency of the control architecture. We observe that the one-third power law controller demands less compensatory action, and therefore lowers the burden on the hardware. Slowing in curved movement regions also unexpectedly allows for faster overall movement.
\end{abstract}

\section{INTRODUCTION}

Can planning locomotion speed according to human-like power laws improve humanoid robot locomotion? Specifically, we examine if the speed of the averaged center of mass reference trajectory affects robot performance. Unfortunately, generated locomotion trajectories may drift from the reference trajectory. A robust control strategy must therefore both correct movement drift and guarantee power law behavior on the actual trajectory. To generate robust power law behavior, we use a globally contracting dynamical system converging to the reference trajectory, regularized to follow power law behavior. To demonstrate our methods,

\footnotetext{
1 are in the Weizmann Institute of Science, Rehovot, Israel \{matan.karklinsky, tamar.flash\}@weizmann.ac.il

2 are with CNRS, LAAS, 7 avenue du colonel Roche, F-31400 Toulouse, France, \{mnaveau, ostasse, soueres\}alaas. fr

${ }^{3}$ is in Hertie Inst., CIN, University Clinic Tübingen, Germany albert. mukovskiy@medizin. uni-tuebingen. de
}

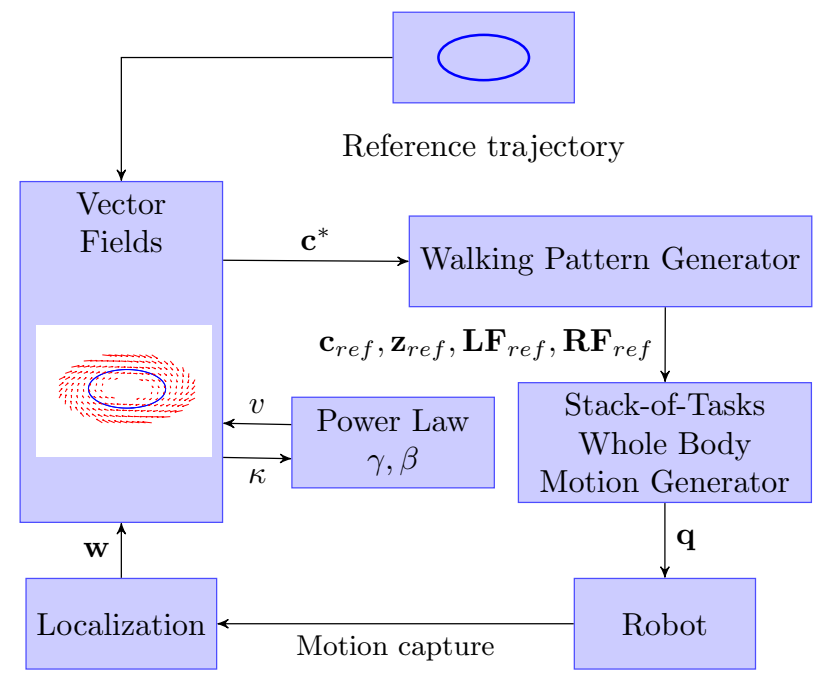

Fig. 1: Power law based closed-loop control. An ellipse reference trajectory is specified and used, together with the power law defined by the velocity gain factor $\gamma$ and $\beta$ exponent constants, to generate the reference vector field. $[x, y, \theta]$ define the position and orientation of a frame attached to the robot's center of mass. The reference vector field defines center of mass velocity $\mathbf{c}^{*}=[\dot{x}, \dot{y}, \dot{\theta}]$ for the walking pattern generator. This particular walking pattern generator provides reference trajectories for the balanced center of mass $\mathbf{c}_{r e f}$, the associated center of pressure $\mathbf{z}_{r e f}$, and the feet $\mathbf{L F}_{r e f}, \mathbf{R F}_{r e f}$. A whole body motion generator uses the reference trajectories to generate a command $\mathbf{q}$ realized by the robot (for HRP-2, this is the configuration vector). The localization component uses position measurements to provide a position $\mathbf{w} \in S E(3)$ of the robot, that again is used as input to the vector field to provide the correcting velocity vector $\mathbf{c}^{*}$. This paper shows that the closed-loop approach reduces motion drifts, but not entirely, and suggests that using human-like power laws may help robots to perform more accurate and more stable motion, while not reducing, or even increasing, movement speed.

we run and analyze dynamic simulations and actual experiments on a HRP-2 robot. For the sake of simplicity, the robot is given an elliptic reference trajectory which obeys one of four distinct power laws.

\section{A. Power laws governing human motion}

The speed of human end effector motion is characterized by the one-third power law behavior; movement speed $v$ decreases when path curvature $\kappa$ increases, following the quantitative relation $v=\gamma \kappa^{-\beta}$, with $\gamma$ the piecewise constant velocity gain factor and $\beta=1 / 3$ the exponent. 
This law, often termed the two-thirds power law due to equivalent formulation determining angular speed $A=\gamma \kappa^{2 / 3}$, was first found for drawing hand motion [21]. It appears for different end effectors under general settings; smooth pursuit eye movements [8], leg motions [19], and even speech [38]. For locomotion along elliptic trajectories, Vielledent et al. found that the center of the head obeyed the one-third power law [40]. Hicheur et al. found that the shoulders' midpoint always obeyed a power law, with different $\beta$ exponents for each walking template [15]. Power law behaviors appear to emerge from jerk minimization [41], [35], [18], [3]. Alternatively, the specific one-third power law, which is equivalent to moving with a constant equi-affine speed [34], [10], may result from equi-affine metrics used by the human brain [11], possibly in a mixture with Euclidean and full affine metrics [2].

Tuned to perception of biological motion, the human visual system perceives one-third power law motion as uniform, rather than movement with constant speed [42]. Coupling between the motor and visual systems in the human brain is supported by stronger and more widespread brain activation patterns [6], and greater event-related desynchronization [25], occurring when subjects watch one-third power law motion, compared with other power law motions. Even imagined movements slow down in curved regions according to the one-third power law [28], [20]. This evidence suggests that humans will perceive a humanoid robot moving according to the one-third power law as more human-like, finding his motions more predictable and easier to follow.

\section{B. Guiding trajectories for humanoid robots}

Planning humanoid robot locomotion is classically accomplished by constructing an optimal sequence of footstep transitions, taken from a discrete set of possibilities, that is kept small to allow reasonable computation time [5], [17]. The limitations of this approach in restricted scenarios yield an alternative approach; planning a reference trajectory which allows generation of the needed contacts [31]. This reference trajectory is usually generated considering balance constraints and geometrical constraints such as feasibility and manipulability. This approach significantly reduces the burden on the motion planner, but raises the demand for controllers capable of finding footsteps or contacts in real time. Planning general contacts is still a very hard problem [9], and finding a center of mass trajectory for a given set of contacts was only recently accomplished [4]. For the restricted case of walking on flat ground, several new approaches [7], [26], [14] allow automatic finding of footstep positions. Therefore, it is now possible to move a humanoid robot by providing only a reference trajectory [26], [14].

In the current study, we attempt to robustly regulate the walking motion on flat ground given a planned reference trajectory. A stable regulation process is important for correcting drifts, that appear due to the interaction of the humanoid robot's soles with the ground [37]. The closed-loop control does reduce the drift. However, in this study we observe that the speed profile of the reference trajectory is crucial; setting a constant reference speed is too naive, and causes a steady-state error in dynamic simulation.

\section{Robust trajectories with contracting dynamics}

Contracting dynamics provide a robust control policy for generating motion patterns; in presence of bounded noise exponential time convergence to a limit cycle or point is guaranteed [22]. By mapping an attractor of a contracting dynamical system to a movement primitive, Giese et al. [13] suggested a general control method for high dimensional systems. In robotics, contraction was already used for robust synchronization of phases of control pattern generators [36] as well as for learning a set of dynamic motion primitives [30]. In this work, we show how contracting dynamics are useful to robustly generate kinematic power law behavior.

\section{REACTIVE WALKING PATTERN GENERATOR}

In order to test power law motions on a humanoid robot we used a recently developed walking pattern generator [26]. This walking pattern generator allows the user to control a humanoid robot as if it was a mobile platform. The input of the walking pattern generator is the velocity $\mathbf{c}^{*}=[\dot{x}, \dot{y}, \dot{\theta}]$ relative to the ground plane, used as a guiding trajectory for the center of mass. The 
walking pattern generator computes automatically the foot $\mathbf{L} \mathbf{F}_{r e f}, \mathbf{R F}_{r e f}$, the center of mass $\mathbf{c}_{r e f}$, and the center of pressure $\mathbf{z}_{r e f}$ trajectories, allowing the robot to maintain two additional constraints during walking: on the placements of foot steps and on the balance. For kinematic and auto-collision reasons, the foot steps are limited to predefined polyhedra. For maintaining balance, the center of mass dynamics is constrained. Assuming a linear inverted pendulum, its associated center of pressure is constrained around the center of the support foot position to maintain balance. Tracking the input velocity by the center of mass has a lower priority than footsteps placement and balancing, therefore the robot creates a swing motion of the center of mass from one foot to another even if the reference velocity has no coronal component. The swinging motion causes perturbations with respect to the reference trajectory [37]. As seen in our dynamic simulations, the swinging motion induces delays and sometimes shifts movement away from the planned reference trajectory. To mitigate the effect of the center of mass swing motion, we considered the barycenter of the robot's feet as the robot's location. This point reflects the robot's position, without the coronal oscillations. In addition to the walking pattern generator, we used a whole body motion generator [23] to compute a motor command realizing the reference trajectory. Finally, two local feedback loops are used to bring back the robot to the reference trajectory; a PID controller over the orientation of the robot, and more importantly a vector field providing the correcting linear velocity for the walking pattern generator. We describe the construction and regularization of this vector field in the next section.

\section{REGULARIZATION OF CONTRACTING OSCILLATORS}

We now describe how to generate power law behavior while correcting for drifts causing deviation from the reference trajectory. This correction is a core function of the closed-loop control system guiding the locomotion of HRP-2 (see Fig. 1).

For a given reference trajectory we design a contracting system; the reference trajectory being a specific solution of the global dynamics. A dynamical system is contracting if all solutions in the contraction region converge exponentially to

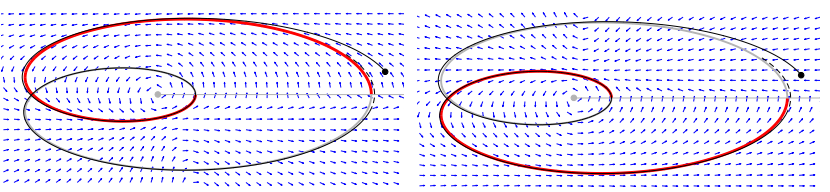

Fig. 2: Polar morphed Andronov-Hopf oscillator with a Limaçon limit cycle. $\phi, \rho$ dynamics define two copies of dynamics for each $x, y$ point.

each other. Then there exists an attractor in this contraction region, and all trajectories in the region converge exponentially to it [22]. Contraction guarantees the exponential decays of perturbations. Partial contraction is contraction towards any of the particular solutions residing inside flow-invariant manifold of the dynamics [33]. For the current work, it suffices that the reference trajectory is an invariant one dimensional submanifold of partially contracting dynamics, with local contraction towards but not along the trajectory. The partial contraction is easy to construct for an arbitrary trajectory, as we demonstrate for cyclic trajectories, the natural test ground of power laws in human motion. We regularize each partially contracting system to obey a power law on all orbits, including the reference trajectory.

\section{A. Polar morphed Andronov-Hopf oscillators}

We recall the construction of a stable dynamical system with desired limit cycle trajectory, a polar morphing of the basic Andronov-Hopf oscillator [29]:

$$
\begin{aligned}
& \dot{\phi}=\omega \\
& \dot{\rho}=\alpha(1-\rho)
\end{aligned}
$$

Defined for $x, y$ Cartesian coordinates of the point in the phase plane as $\rho=\frac{1}{r^{2}}=\frac{1}{x^{2}+y^{2}}, \phi=$ $\tan ^{-1}\left(\frac{y}{x}\right)$ describing the radial and the angular dynamics, and $\omega, \alpha>0$ constants. It is partially contracting, since the Jacobian of the $\rho$ subsystem is uniformly negative definite $J_{\rho}<-\alpha<0$. For any reference path of the limit cycle attractor, $r_{0}(\phi)$, given in polar coordinates, this oscillator can be morphed [1]:

$$
\begin{aligned}
\dot{\phi} & =\omega \\
\dot{\rho} & =\alpha(F(\phi)-\rho)+\omega \frac{d F}{d \phi}
\end{aligned}
$$


It is still partially contracting, with $F(\phi)=\frac{1}{r_{0}^{2}(\phi)}$ depending on the limit cycle shape. Interestingly, we may choose the range of $\phi$ to be $[0,2 \pi n)$ for any integer $n$, not just $n=1$. This allows a limit cycle with winding number $n$, which may selfintersect when viewed in $(x, y)$ plane (see Fig. 2). The dynamics defined in the $(\phi, \rho)$ plane map to dynamics of $(x, y)$ plane through a covering map; each point $(x, y)$ in the punctured plane has $n$ copies of dynamics, one surrounding each of its polar angles $\phi^{i} \in[2 \pi(i-1), 2 \pi i)$. To run the dynamics on the $(x, y)$ plane, keeping track of the winding of $\phi$ is needed.

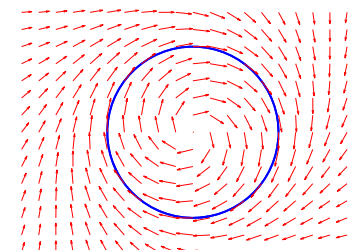

(a)

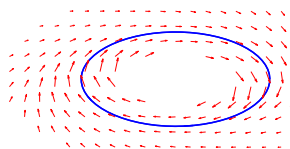

(c)

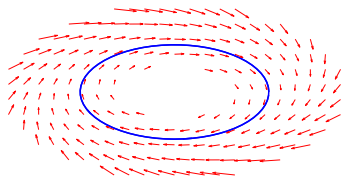

(e)

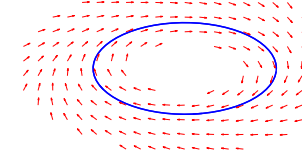

(b)

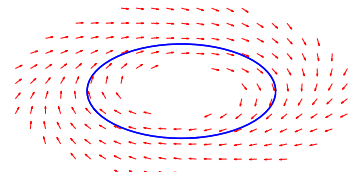

(d)

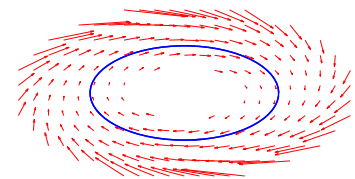

(f)
Fig. 3: Morphing and regularization of an Andronov-Hopf oscillator. a) The unit cycle oscillator. b) an elliptic morphing. c)-f) Four power law regularizations according to $\beta=-1 / 3,0,1 / 3,2 / 3$ power laws respectively. Each regularization keeps directions but changes speeds of the elliptic vector field.

\section{B. Temporal regularization of a dynamical system}

In this section, we define the power law regularization of dynamical systems and examine the conditions for its applicability. Under reasonable conditions, the regularized dynamical system has exponential convergence to the limit cycle of the original system. For some special cases the regularized system is contracting.

1) Power law regularization: We focus on regularization with a power law $v=h(\kappa)=\gamma \kappa^{-\beta}$, with $\gamma$ a global constant and $\beta$ the exponent value. This is the basic most useful example of the general
Euclidean invariant dependency of speed upon path geometry, $v=h\left(\kappa, \frac{d \kappa}{d s}, \ldots, \frac{d^{n} \kappa}{d s^{n}}\right)$ (see [2]).

Definition. For a dynamical system $\dot{\boldsymbol{x}}=F(\boldsymbol{x})$, and a power law $v=h(\kappa)$, we define the $h$ regularization of $F$, denoted by $\dot{\boldsymbol{x}}=F_{h}(\boldsymbol{x})$, as $F_{h}(\boldsymbol{x})=\frac{F(\boldsymbol{x})}{|F(\boldsymbol{x})|} h(\kappa(\boldsymbol{x}))$, with $\kappa$ the curvature of the orbit at point $\boldsymbol{x}$.

At each point, the orbit of $F_{h}$ geometrically coincides with the orbit of $F$. Additionally, the speed along each orbit of $F_{h}$ satisfies the law $h$. Each velocity vector in the regularized system $F_{h}$ has the same direction, but not necessarily the same size, as the matching velocity vector in the original system $F$. Our power laws are positively monotone so the directions of flows of $F$ and $F_{h}$ match.

Assumptions. We consider a region $U$ which is a compact trapping region in the plane for $F$ without fixed points. We require that for $F$ 's orbits $\kappa$ is defined and bounded, and therefore $h$ is defined and bounded; for each $\boldsymbol{x} \in U, h(\boldsymbol{x}) \geq C_{1}>0$ for some global constant $C_{1}$. We require that $F$ has bounded speed $|F(\boldsymbol{x})|<C_{2}$ for some global constant $C_{2}$.

These assumptions guarantee that, if $F$ is contracting in $U$ to some limit cycle, then each solution converges globally exponentially to this limit cycle in $U$ (see Lohmiller and Slotine's [22], Theorem 1 ). While we do not claim that $F_{h}$ is generally contracting, our assumptions yield global exponential convergence of $F_{h}$ to the limit cycle of $F$. This is true because $\left|F_{h}(\mathbf{x})\right|=\frac{h(\mathbf{x})}{|F(\mathbf{x})|}|F(\mathbf{x})| \geq \frac{C_{1}}{C_{2}}|F(\mathbf{x})|$. The limit cycle of $F_{h}$ is geometrically identical to that of $F$, and movement on it obeys the $h$ power law.

2) Curvature of the orbits of the circular Andronov-Hopf oscillator: Power law regularization has singularities in inflection points and along straight trajectories; if $\kappa=0$ and $\beta>0$, the power law speed is infinite. This can be overcome in two ways. For most practical needs the power law speed $v$ can be bounded; Viviani and Stucchi [42] defined $v=\gamma(\kappa+\alpha)^{-\beta}$, with some constant $\alpha>0$ preventing the singularity at $\kappa=0$. Alternatively, we can restrict the discussion to regions of positive curvature. For the morphed AndronovHopf oscillators, around a planned cyclic trajectory 
with positive curvature, there exists a band of bounded nonzero curvature, guaranteeing that the regularization process will result in finite speeds.

The dynamics of $\rho(\phi)$ define $\kappa(\rho)$, the curvature of the orbit. For circular Andronov-Hopf oscillators, the dynamics are invariant with respect to rotations around the origin $(x, y)=0$. Therefore local curvature of the vector flow $\kappa(\rho)$ is a function of $\rho$ only, independent of $\phi$. For any orbit, its local curvature $\kappa(\rho)$ equals zero exactly where it crosses a circle $\rho=\rho_{\kappa=0}$, concentric to the unit circle that is the limit cycle, but with larger radius. Therefore, in the circular band $C \geq \rho \geq \rho_{\kappa=0}+\epsilon$, for arbitrarily small $\epsilon$ and arbitrarily large $C$, the curvature is strictly positive $\kappa \geq C_{1}>0$, speed is bounded, and our assumptions hold, allowing power law regularization that results in exponential convergence to the limit cycle.

3) Power law regularized circular oscillator is contracting: For the unit circle Andronov-Hopf oscillator, the power law function depends on curvature which is independent of $\phi$. Denoting $\tilde{\rho}=\rho-1=\frac{1}{r^{2}}-1$ and $h(\tilde{\rho})$ the regularization power law function. $h(\tilde{\rho})>0$ in the area where the curvature is strictly positive, and the regularized dynamics are:

$$
\begin{aligned}
& \dot{\tilde{\rho}}=-\tilde{\rho} h(\tilde{\rho}) \\
& \dot{\phi}=\omega g(\tilde{\rho})
\end{aligned}
$$

With $g(\tilde{\rho})=\frac{h(\tilde{\rho})}{r}$. The $\tilde{\rho}$ dynamics are independent of $\phi$. The Jacobian of the $\tilde{\rho}$ subsystem is $J=$ $-\left(h(\tilde{\rho})-\tilde{\rho} h^{\prime}(\tilde{\rho})\right)$, and after the local coordinate change $\Theta(\tilde{\rho})=\frac{1}{h(\tilde{\rho})}$ (see [22]) it is:

$$
\begin{aligned}
J_{\Theta} & =(\dot{\Theta}+\Theta J) \Theta^{-1}= \\
& =\left(\frac{h^{\prime} \tilde{\rho}}{h}+\frac{1}{h}\left(-h-\tilde{\rho} h^{\prime}\right)\right) h \\
& =-h<0
\end{aligned}
$$

The dynamics of $\tilde{\rho}$ are therefore contracting. The dynamics of $\phi$ are bounded in $\tilde{\rho}$ and therefore it admits the nonlinear local $\tilde{\rho}$ dependent coordinate system where $\phi$ is indifferent, similar to the original oscillator [22].

4) A contracting one-third power law regularized elliptic oscillator: For the one-third power law, any elliptic system generated as a linear transformation of the regularized unit circle oscillator is
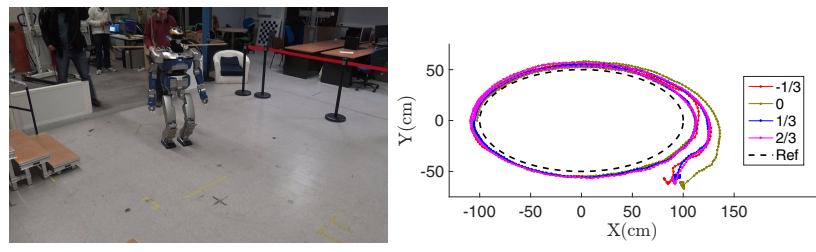

Fig. 4: Experimental setup, the robot walks according to a reference ellipse, obeying one of four different power law speeds. Trajectories for each power law converge around a limit cycle ellipse.

contracting. The argument of the unit circle regularized oscillator, based on the circular symmetry of $\kappa$, holds for uniform scaling. A global equiaffine transformation of the plane conserves the one-third power law and therefore the transformation of the unit circle dynamics to elliptic dynamics using the combination of a scaling and an equiaffine transformation conserves contraction of the regularized system.

\section{EXPERIMENTAL METHODS}

\section{A. Dynamic simulations}

We simulated motion using the OpenHRP simulator, that computes the contact forces and HRP2's rigid body mechanics, and includes a model of the compliance of the robot ankles. We implemented the control architecture depicted in Fig. 1 in OpenHRP. The elliptic reference trajectory, with radii sized $1 \mathrm{~m}$ and $0.5 \mathrm{~m}$, was assigned four power laws, $v=\gamma \kappa^{-\beta}$, with exponents $\beta=$ $-1 / 3,0,1 / 3,2 / 3$ and $\gamma$ adjusted to always make reference lap time identical; 2 minutes. We analyzed the trajectories of the center of mass using MATLAB. Because the discussed power laws refer to averaged center of mass trajectories, we removed the coronal swing motion by applying a procedure for finding the middle points of each sway. Each middle point was the average of two consecutive local signed curvature extrema with opposite signs, with time defined as the average of the times of these two points. We used the middle points trajectory for all analysis purposes. We estimated the power law exponents $\beta$ using nonlinear regression [24]. Repeating the procedure with log-log linear regression yielded similar $\beta$ values. Speed was extracted from the middle points trajectory 


\begin{tabular}{||c||cc|c|c||}
\hline \hline SIM & & & & \\
Ref. $\beta$ & Sim. $\beta$ & $R^{2}$ & RMSD (cm) & T (s) \\
\hline-0.33 & -0.21 & 0.46 & 5.2 & 140.0 \\
0 & 0.05 & 0.27 & 3.0 & 134.0 \\
0.33 & 0.33 & 0.99 & 0.2 & 130.0 \\
0.67 & 0.60 & 0.94 & 0.4 & 127.2 \\
\hline \hline EXP & & & & \\
Ref. $\beta$ & Exp. $\beta$ & $R^{2}$ & RMSD (cm) & T (s) \\
\hline-0.33 & -0.23 & 0.70 & 7.3 & 150.4 \\
0 & 0.02 & 0.03 & 5.5 & 142.4 \\
0.33 & 0.34 & 0.79 & 6.3 & 134.4 \\
0.67 & 0.57 & 0.90 & 6.8 & 133.6 \\
\hline \hline REF & - & - & 0 & 120.0 \\
\hline \hline
\end{tabular}

TABLE I: Results from dynamic simulation (SIM) and actual robot experiment (EXP); for different reference power laws (Ref. $\beta$ ), simulated power law exponent (Sim. $\beta$ ) and actual motion power law exponent (Exp. $\beta$ ) calculated using nonlinear regression [24] with $\mathrm{R}$ squared error $\left(R^{2}\right)$, root mean squared distance to the reference path (RMSD) and duration (T) of the generated ellipse trajectory are given, with those of the reference trajectory (REF). Geometrically, in simulation the one-third power law, $\beta=1 / 3$, is most exact, and in experiment the constant speed was more exact. Temporally, despite identical reference times, higher $\beta$ exponents yield faster motions for both simulation and experiment, but always slower than the reference time.

using a noise-insensitive filter [16]. Curvature was extracted from the reference ellipse.

\section{B. Experiments on HRP-2}

We reproduced the simulated movements on the actual HRP-2 robot; the robot started standing approximately $60 \mathrm{~cm}$ behind the tip of the ellipse, and walked until completing two laps, guided by the dynamical system. We used the linearly morphed contracting elliptic dynamics, regularized by each power law, repeating $\beta$ and $\gamma$ values used in simulations. We measured center of mass trajectories using the motion capture system, low pass filtered them at $0.1 \mathrm{~Hz}$, and then analyzed them identically to the simulated trajectories.

\section{RESULTS}

To evaluate the effect of applying the one-third power law to humanoid robot walking, we present dynamic simulations and real robot experiments testing elliptic motions generated by the walking pattern generator (Fig. 1). We compared four power laws, with exponents $\beta=-1 / 3,0,1 / 3,2 / 3$.

\section{A. Dynamic simulation results}

1) Power law patterns are reproduced: The $\beta$ values of simulated motions reflected the $\beta$ values of the reference speed profiles, that were noisily

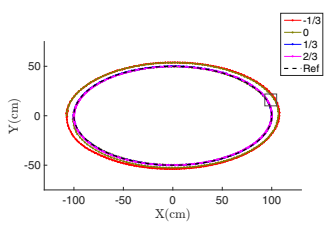

(a)

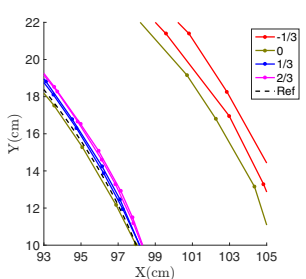

(b)
Fig. 5: a) Dynamic simulation paths, with different reference speeds. b) Zoom in on the box in plot a. Simulations with positive $\beta=1 / 3,2 / 3$ drift less than $0.5 \mathrm{~cm}$ from the reference path. Simulations with constant speed and negative power law $\beta=0,-1 / 3$ drift outside of the reference elliptic path.

reproduced (Fig. 6, left). The simulation yielded positions of maximal speed slightly shifted with respect to maximal speed positions predicted by the power law based on the curvature of the actual path; for $\beta=-1 / 3,1 / 3,2 / 3$ shifts along the trajectory, of $22,4,-15 \mathrm{~cm}$ of the simulated with respect to reference speed peaks, occurred. Unpredictably, constant speed $\beta=0$ power law yielded an oscillatory curvature-dependent speed profile.

2) Drift correction by one-third power law: As predicted, the simulated one-third power law resulted in reduced drift compared to constant speed and other power laws. For $\beta=1 / 3$ the path was most similar to the reference path, with next best being the $\beta=2 / 3$ power law. The constant speed $\beta=0$ and $\beta=-1 / 3$ yielded drifts; the simulated elliptic paths were larger than the reference elliptic path (see table I and Fig. 5). Interestingly, the constant speed $\beta=0$ path deviated from the reference frame gradually and not immediately upon movement initiation (Fig. 5.b).

3) Increase in $\beta$ exponent decreases duration: Simulated motions took more time than the reference time; always 2 minutes per lap. Surprisingly, the exponent $\beta$ affected simulated movement duration; the higher the $\beta$ the faster the motion, with duration closer to the reference time (table I).

\section{B. Experimental results}

1) Power law patterns are reproduced: The reference speed power laws were noisily reproduced by the robot (see table I for $\beta$ values and their $R^{2}$ errors, and Fig. 6, right for speed profiles).

2) Geometrical drift does not fully vanish: Overall, experimental results showed larger drifts 


\begin{tabular}{||c||c|c|c|c||}
\hline \hline Ref. $\beta$ & -0.33 & 0 & 0.33 & 0.67 \\
\hline Norm $(\mathrm{m})$ & 1.416 & 0.950 & 0.642 & 1.124 \\
Orientation $(\mathrm{deg})$ & 76.83 & 89.60 & 60.45 & 77.28 \\
Force $(k N \times \mathrm{s})$ & 21.93 & 23.54 & 19.80 & 21.01 \\
\hline
\end{tabular}

TABLE II: Analysis of the odometry frame and forces. For each of the four power laws the distance from center (Norm), body orientation (Orientation) and integrated norm tangential force measured on ankle (Force) are given. The one-third power law is outperforming all other power laws.

than simulation. The constant reference speed resulted in the lowest drift (table I), outperforming the positive $\beta$ power laws. Different convergence trajectories (Fig. 4), may arise from slightly different initial positioning of the robot.

3) Increased $\beta$ decreases duration: Actual robotic movement took longer time than simulation. In the experiment, as in simulation, despite having the same reference time, higher $\beta$ values yielded faster movements.

4) Reduced controller corrections: To estimate the feedback correction produced by the vector field and the PID controllers, we analyzed the internal odometry frame of the walking pattern generator. We examined the last crossing point of the $x$ axis by each center of mass trajectory. We measured deviation from the baseline, the planned position and orientation, to gain error values reflecting the accumulated error along the two laps, caused by sliding. Additionally, we examined tangential forces on the ankle, indicating the amount of compensated sliding. The one-third power law showed smaller accumulated errors, compared to the other power laws (table II). Less error needing compensation implicates lower burden on the hardware.

\section{CONCLUSIONS}

In simulation and experiment, we tested how stable generation of power laws may help humanoid robot walking. The simulations and experiments reproduced the reference power law's temporal patterns. As predicted, the one-third power law, used by humans, appears beneficial; it reduced drift in simulations and also decreased the need for sliding compensation in actual robot motion; the closed-loop controller managed to produce movement with any of the four tested power laws, but maintaining a one-third power law lowered energy expenditure. Surprisingly, our results suggest that
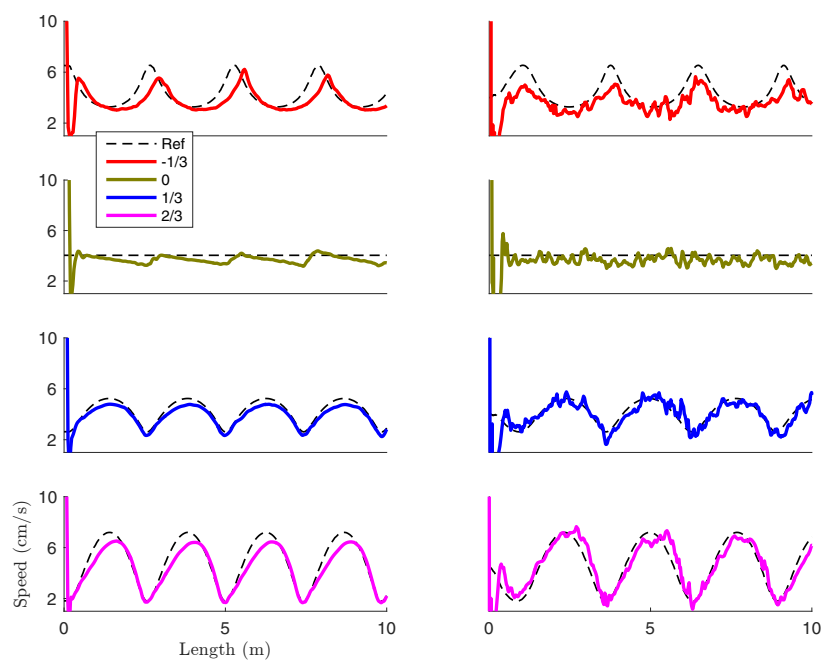

Fig. 6: Speed profiles for dynamic simulations (Left) and robot experiment (Right), with four different reference speeds (Ref). Both simulated and actual robot motions reproduce the spatio-temporal power law patterns.

using higher $\beta$ exponents power laws may allow for faster movements.

We exemplified, studying elliptic motions, the importance of using path-adjusted speed profiles. Selecting optimal speed profiles for reference center of mass trajectories of planar robotic locomotion is not fully solved. Different perspectives describing human motion; optimization [12], [39] or geometric invariance [11], [2], may provide inspiration for a solution.

Except in locomotion, we suggest two additional applications of speed modulation according to the geometry of the reference trajectory. First, to use power laws as a constraint for kino-dynamic motion planning [32]. Second, to find bounds on curvature for planning free-flyer motion [27].

\section{ACKNOWLEDGMENTS}

Financial support by the European Commission within the FP7 project KoroiBot (No. 611909 ICT2013.2.1 Cognitive Systems and Robotics) is gratefully acknowledged. We thank Jindrich Kodl for suggesting a sway removal procedure. We thank the anonymous reviewers for fruitful suggestions.

\section{REFERENCES}

[1] M. Ajallooeian, J. van den Kieboom, A. Mukovskiy, M.A. Giese, and A.J. Ijspeert. A general family of morphed nonlinear phase oscillators with arbitrary limit cycle shape. Physica D: Nonlinear Phenomena, 263:41-56, 2013.

[2] D. Bennequin, R. Fuchs, A. Berthoz, and T. Flash. Movement timing and invariance arise from several geometries. PLoS Comp. Biol., 5(7):e1000426, 2009. 
[3] A. Biess. Shaping of arm configuration space by prescription of non-Euclidean metrics with applications to human motor control. Phys. Rev. E, 87(1):012729, 2013.

[4] J. Carpentier, S. Tonneau, M. Naveau, O. Stasse, and N. Mansard. A versatile and efficient pattern generator for generalized legged locomotion. In IEEE/RAS Int. Conf. on Robotics and Automation, 2016.

[5] J. Chestnutt. Motion Planning for Humanoid Robots, chapter Navigation and gait planning. Springer Verlag, 2010.

[6] E. Dayan, A. Casile, N. Levit-Binnun, M.A. Giese, T. Hendler, and T. Flash. Neural representations of kinematic laws of motion: evidence for action-perception coupling. P.N.A.S., 104(51):20582-20587, 2007.

[7] R. Deits and R. Tedrake. Footstep planning on uneven terrain with mixed-integer convex optimization. In IEEE/RAS Int. Conf. on Humanoid Robotics (ICHR), 2014.

[8] C. de'Sperati and P. Viviani. The relationship between curvature and velocity in two-dimensional smooth pursuit eye movements. J. of Neurosci., 17(10):3932-3945, 1997.

[9] A. Escande, A. Kheddar, and S. Miossec. Planning contact points for humanoid robots. Robotics and Autonomous Systems, 61(5):428 - 442, 2013.

[10] T. Flash and A.A. Handzel. Affine differential geometry of human arm trajectories. Abs. of the Soc. for Neurosci., 1996.

[11] T. Flash and A.A. Handzel. Affine differential geometry analysis of human arm movements. Bio. cybernetics, 96(6):577601, 2007.

[12] T. Flash and N. Hogan. The coordination of arm movements: an experimentally confirmed mathematical model. $J$. of Neurosci., 5(7):1688-1703, 1985.

[13] M.A. Giese, A. Mukovskiy, A. Park, L. Omlor, and J.E. Slotine. Real-time synthesis of body movements based on learned primitives. In Cremers D, et al. (eds): LNCS 5604, 5604:107-127, 2009. reviewed.

[14] A. Herdt, N. Perrin, and P.B. Wieber. Walking without thinking about it. In IEEE/RSJ Int. Conf. on Intelligent Robots and Systems (IROS), pages 190-195, 2010.

[15] H. Hicheur, S. Vieilledent, M.J.E. Richardson, T. Flash, and A. Berthoz. Velocity and curvature in human locomotion along complex curved paths: a comparison with hand movements. Exp. Brain Res., 162(2):145-154, 2005.

[16] P. Holoborodko. Smooth noise robust differentiators, 2008.

[17] A. Hornung, A. Dornbush, M. Likhachev, and M. Bennewitz. Anytime search-based footstep planning with suboptimality bounds. In IEEE/RAS Int. Conf. on Humanoid Robotics (ICHR), pages 674-679, 2012.

[18] D. Huh and T.J. Sejnowski. Spectrum of power laws for curved hand movements. P.N.A.S., 112(29):E3950-E3958, 2015.

[19] Y.P. Ivanenko, R. Grasso, V. Macellari, and F. Lacquaniti. Two-thirds power law in human locomotion: role of ground contact forces. Neuroreport, 13(9):1171-1174, 2002.

[20] M. Karklinsky and T. Flash. Timing of continuous motor imagery: the two-thirds power law originates in trajectory planning. J. of Neurophys., 113(7):2490-2499, 2015.

[21] F. Lacquaniti, C. Terzuolo, and P. Viviani. The law relating the kinematic and figural aspects of drawing movements. Acta Psychologica, 54(1-3):115-130, 1983.

[22] W. Lohmiller and J.E. Slotine. On contraction analysis for non-linear systems. Automatica, 34(6):683-696, 1998.

[23] N. Mansard, O. Stasse, P. Evrard, and A. Kheddar. A versatile generalized inverted kinematics implementation for collaborative working humanoid robots: The stack of tasks. In Int. Conf. on Adv. Robotics (ICAR), page 119, 2009.
[24] U. Maoz, E. Portugaly, T. Flash, and Y. Weiss. Noise and the two-thirds power law. In $A d v$. in Neural Information Processing Systems, pages 851-858, 2005.

[25] Y. Meirovitch, H. Harris, E. Dayan, A. Arieli, and T. Flash. Alpha and Beta Band Event-Related Desynchronization Reflects Kinematic Regularities. J. of Neurosci., 35(4):16271637, 2015.

[26] M. Naveau, M. Kudruss, O. Stasse, C. Kirches, K. Mombaur, and P. Soueres. A reactive walking pattern generator based on nonlinear model predictive control. IEEE Robotics and Automation Letters, 2016. accepted.

[27] A. Orthey. Exploiting Structure in Humanoid Motion Planning. PhD thesis, Univ. Toulouse III - Paul Sabatier, France, 2015.

[28] C. Papaxanthis, C. Paizis, O. White, T. Pozzo, and N. Stucchi. The relation between geometry and time in mental actions. PloS one, 7(11):e51191, 2012.

[29] A. Park, A. Mukovskiy, J. E. Slotine, and M.A. Giese. Design of dynamical stability properties in character animation. In VRIPHYS, pages 85-94, 2009.

[30] B.E. Perk and J.E. Slotine. Motion primitives for robotic flight control. CoRR, abs/cs/0609140, 2006.

[31] N. Perrin, O. Stasse, F. Lamiraux, and E. Yoshida. Weakly collision-free paths for continuous humanoid footstep planning. In IEEE Int. Conf. on Intelligent Robots and Systems (IROS), pages 4408-4413, 2011.

[32] Q. Pham, S. Caron, and Y. Nakamura. Kinodynamic planning in the configuration space via admissible velocity propagation. In Robotics System and Science, 2009.

[33] Q. Pham and J. E. Slotine. Stable concurrent synchronization in dynamic system networks. Neural Networks, 20(1):62-77, 2007.

[34] F.E. Pollick and G. Sapiro. Constant affine velocity predicts the $1 / 3$ power law of planar motion perception and generation. Vision Res., 37(3):347-353, 1997.

[35] M.J.E. Richardson and T. Flash. Comparing smooth arm movements with the two-thirds power law and the related segmented-control hypothesis. J. of Neurosci., 22(18):82018211, 2002.

[36] K. Seo, S. Chung, and J. E. Slotine. CPG-based control of a turtle-like underwater vehicle. Autonomous Robots, 28(3):247-269, 2010.

[37] O. Stasse, A.J. Davison, R. Sellaouti, and K. Yokoi. Realtime $3 \mathrm{~d}$ slam for humanoid robot considering pattern generator information. In IEEE/RSJ Int. Conf. on Intelligent Robots and Systems (IROS), pages 348-355, 2006.

[38] S.M. Tasko and J.R. Westbury. Speed-curvature relations for speech-related articulatory movement. J. of Phonetics, 32(1):65-80, 2004.

[39] E. Todorov and M.I. Jordan. Optimal feedback control as a theory of motor coordination. Nature Neurosci., 5(11):1226$1235,2002$.

[40] S. Vieilledent, Y. Kerlirzin, S. Dalbera, and A. Berthoz. Relationship between velocity and curvature of a human locomotor trajectory. Neurosci. Letters, 305(1):65 - 69, 2001.

[41] P. Viviani and T. Flash. Minimum-jerk, two-thirds power law, and isochrony: converging approaches to movement planning. J. of Exp. psychology. Human perception and performance, 21(1):32-53, 1995.

[42] P. Viviani and N. Stucchi. Biological movements look uniform: evidence of motor-perceptual interactions. J. of Exp. psychology. Human perception and performance, 18(3):603623, 1992. 